\title{
Survey of Nursing Perceptions of
} Medication Administration Practices, Perceived Sources of Errors and Reporting Behaviours

Markirit Armutlu, Mary-Lou Foley, Judy Surette, Eric Belzile and Jane McCusker

\section{Abstract}

In January 2003, St. Mary's Hospital Center in Montreal, Quebec, established an interdisciplinary Committee on the Systematic Approach to Medication Error Control to review the whole process of medication administration within the hospital and to develop a systematic approach to medication error control. A cross-sectional survey on medication administration practices, perceived sources of errors and medication error reporting of nurses, adapted from a nursing practice survey and medication variance report (Sim and Joyner 2002), was conducted over a two-week period in February 2004. The results were analyzed by years of experience (greater or less than five years) and patient care unit of practice. The perceived source of error most often cited was transcription (processing), and the second most frequently cited source was the legibility of handwritten medication orders (prescribing).

The results demonstrate no significant difference in medication safety practices or in perceptions of errors by years of experience. Nurses appear to adapt to the safety culture of the unit rather quickly, certainly within their first five years on the unit. Good medication error reporting behaviour was noted, with no differences between all comparative groups within both years of experience and unit of practice. Quality improvement initiatives to improve the safety of medica- tion administration practices have included the development of a nursing medication administration handbook, the revision of policies and procedures related to medication administration safety, the standardization of solutions and limited variety of high-risk medication dosages and the reduction of handwritten reorders. The need for ongoing education and information sessions on policies and procedures specific to safe medication practices for

all nurses, regardless of years of experience, was identified. 
t. Mary's Hospital Center, located in Montreal, Quebec, is a 316-bed acute care, university-affiliated community hospital, with 65 beds designated for long-term care and a very large outpatient and family medicine program. The hospital has approximately 1,800 full-time and part-time employees, including about 618 registered nurses. In addition, there are roughly 254 active and 104 associate physicians practising at St. Mary's Hospital. The hospital provides a wide variety of acute, critical, emergency, cancer, mental health, maternal-child, surgical, medical care and diagnostic services, in addition to an active clinical teaching program.

The medication administration process, including the reporting and management of medication errors, was identified in 2002 by the hospital's Quality and Risk Management Coordinating Committee as a priority area for quality improvement. Starting in the spring of 2002, in-service education sessions were offered to every nurse on every shift to encourage the reporting of all adverse healthcare services-related events and, in particular, medication errors. In 2003-2004, $20 \%$ of all reported incidents and accidents were medication related; this percentage has fluctuated between 22 and $26 \%$ over the past three years. In 2003-2004, 732,902 medication orders were processed and administered; this increased to 767,829 (a 4.8\% increase) by 2006-2007.

In January of 2003, a Committee on the Systematic Approach to Medication Error Control was established and mandated to review the whole process of medication administration within the hospital and to develop a systematic approach to medication error control, as outlined by Anderson and Webster (2001) and Kohn et al. (1999). This committee was composed of a physician, a nurse, a nurse manager, the chief pharmacist and a quality analyst. As part of this mandate, a cross-sectional survey of nurses on medication administration practices was conducted in February 2004. The objectives of the survey were to (1) describe nurses' perceptions of medication administration practices, reporting of errors and sources of medication errors and (2) describe the relationships between nurses' perceptions and their years of experience and the patient care unit on which they worked.

Prior research has addressed responses to observed medication errors (Kazaoka 2007; McBride-Henry and Foureur 2006; Nicholson 2006), incident reporting (Cohen et al. 2003; Mayo and Duncan 2004), safety practices in medication administration (Cohen et al. 2003; O'Shea 1999; Sim and Joyner 2002; Winson 1991), perceived sources of errors (Hicks et al. 2004; Leape et al. 1995; Mayo and Duncan 2004; Osborne 1999) and the impact of mentorship programs on new nurses (Casey et al. 2004; Duchscher and Cowin 2006; Stewart and Kreuger 1996; Thomka 2001; Thomka 2007). Literature has shown that senior nurses, as preceptors, may take risks as a result of their comfort or familiarity with the medication process, and junior nurses, as "preceptees," may feel pressured to emulate the practices of the senior nurses, thus quickly adopting and fitting into the culture of the patient care unit (Thomka 2007). To our knowledge, no studies have examined both nursing perceptions and medication safety practices by years of experience.

\section{Methods}

All registered nurses regardless of status or shift and in all patient care areas where nurses administer medications were asked to participate. Nurse managers, supervisors, liaison nurses and nurses working in areas where no medications are administered were excluded from the study. From the initial total of 618 registered nurses, 508 qualified for the study. Of these, 122 were excluded as they were on a leave of absence, sick leave or vacation, unavailable, participated in the pretest or refused. The final number of questionnaires distributed was 386 and, of these, 205 were returned. The information collected was nonnominative, ensuring the anonymity of respondents.

In December 2003, the survey was pretested in a focus group consisting of 10 members of the Professional Nurses Practice Committee (PNPC). Based on their feedback, the survey was revised to improve clarity and ensure anonymity of the respondents. Over a two-week period in February 2004, all unit PNPC representatives were given questionnaires to distribute in their own patient care unit or clinical area. All nurses were approached at work to complete the survey. The PNPC representative recorded distribution data. A self-addressed return envelope was attached to each questionnaire. Each unit or area was canvassed several times over a two-week period to maximize distribution over weekends and all shifts.

The questionnaire consisted of multiple-choice and openended questions (Figure 1) directed toward current practice, perceptions of sources of error, error reporting practices and demographic information. Eight questions on current practice were adapted and expanded from a seven-question medication administration practice survey developed by a community hospital in the United States (Sim and Joyner 2002). Possible responses were always, frequently, about half the time, rarely and never. For the analyses, these responses were coded from one to five, respectively, for good/favourable medication practices (questions 1, 3, 4, 5, 7 and 8); responses for two questions (questions 2 and 6; see Figure 1) were reverse-coded for poor/ unfavourable practices. Medication error reporting behaviour was examined through the use of a single multiple-choice question with a five-point response scale from always to never.

Nurses were then asked to select what they perceived as the six most common sources of error from a checklist of 16 specific causes of errors. They also had the opportunity to list three other causes of errors. When possible, the other causes of errors mentioned by nurses were recoded into the 16 specified causes within the survey. The perceived sources of medication errors 
Figure 1. Medication administration survey In an effort to understand the current medication practices, this survey is being circulated to all the RNs 0

\section{Current Practice}

Thinking of the medications that you have administered during the last month, how frequently:

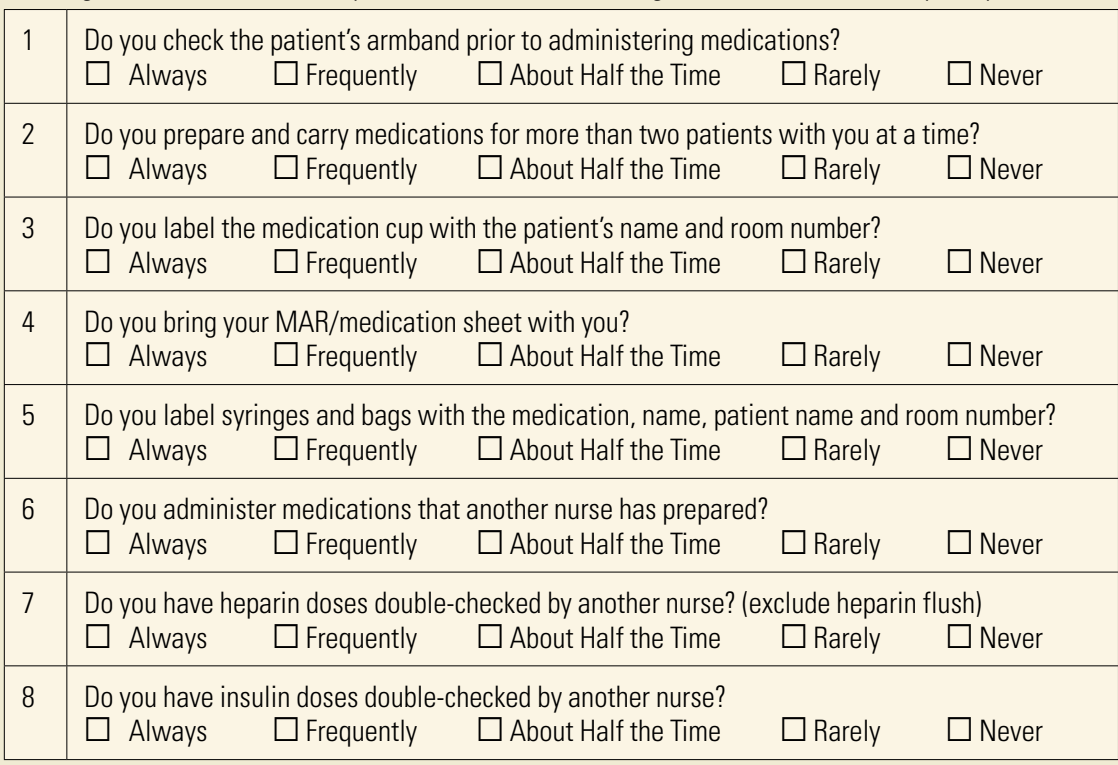

Sources of Error: In your opinion, what are the six main causes of error? Please check only 6 of the following:

\begin{tabular}{|c|c|c|c|}
\hline$\square$ & a) computer entry error (processing) & $\square$ & k) miscalculation (administering) \\
\hline$\square$ & b) transcription error (processing) & $\square$ & I) procedure/policy not followed (administering) \\
\hline$\square$ & c) no order sent to/received by pharmacy (processing) & $\square$ & m) filled incorrectly (dispensing) \\
\hline$\square$ & d) error checking MAR/medication sheet (processing) & $\square$ & n) mislabelled (dispensing) \\
\hline$\square$ & e) legibility (prescribing) & $\square$ & o) names of meds look alike (dispensing) \\
\hline$\square$ & f) incorrect order (prescribing) & $\square$ & p) labels of meds look alike (dispensing) \\
\hline$\square$ & g) confusing order/instructions (prescribing) & $\square$ & q) other, specify: \\
\hline$\square$ & h) misunderstood verbal order (prescribing) & $\square$ & r) other, specify: \\
\hline$\square$ & i) knowledge deficit (administering) & $\square$ & s) other, specify: \\
\hline$\square$ & j) distractions (administering) & & \\
\hline
\end{tabular}

Reporting Errors: I report medication errors using the Incident/Accident form (AH223). Please check one response.
$\square \quad$ Always
$\square \quad$ Frequently
$\square$ About Half the Time
$\square \quad$ Rarely
$\square \quad$ Never

Demographics: Please indicate the area where you usually work:

\begin{tabular}{|l|l|l|l|l|l|}
\hline$\square$ & Psychiatry / OPD Clinic & $\square$ & 5 Main/ONC Clinic & $\square$ & 5 North \\
\hline$\square$ & Family Medicine Center & $\square$ & 7 Main & $\square$ & 8 Main \\
\hline$\square$ & 6 North/6 South & $\square$ & 5 South & $\square$ & Dialysis \\
\hline$\square$ & 4 Main/Women's CI/Nursery/Case room & $\square$ & ICU/CCU/MDC & $\square$ & RR/SDC/OR \\
\hline$\square$ & Emergency & & & & \\
\hline
\end{tabular}

Years of practice as RN: $\square \quad 5$ or less $\square$ more than 5

CCU = Coronary Care Unit; ICU = Intensive Care Unit; MAR = medication administration record; MDC = Medical Day Center; ONC Clinic = Oncology Clinic; OPD Clinic = Out-Patient Clinic; OR = Operating Room; PNPC = Professional Nurses Practice Committee; RN = Registered Nurse; RR = Recovery Room; SDC = Surgical Day Center; Women's Cl = Women's Clinic. 
were then grouped into five categories: processing (four specific causes), prescribing (four causes), administering (four causes), dispensing (four cause) and other (three causes). The proportions of errors in the first four categories were calculated for each nurse as the sum of errors in that category divided by the total potential causes in the category.

Further details about the methods used are presented in Appendix A.

\section{Results}

Among the 205 questionnaires received, 144 with complete data on medication practices, years of experience and hospital unit were retained for the principal components analysis (Table 1). Two factor solutions emerged from the principal components analysis, named "dosage care" (with three items) and "right patient" (with four items), and two single items assessed "preparing/carrying medications" and "error reporting."

"Error reporting" had the lowest mean (1.7 on a scale from one to five), indicating the most favourable medication practice (Table 2). At the other extreme, "preparing/carrying medications" showed the worst level (mean of 3.5). Work unit was significantly associated with "dosage care," "right patient" and "preparing/carrying medications," whereas years of experience was not associated with any of the four behaviour scales. After applying Bonferroni correction, "dosage care" had more favourable scores in the maternal-child and surgical units and less favourable scores in the critical care, emergency and medical units. On the other hand, "right patient" had significantly more favourable scores in the emergency, medical and surgical units and less favourable scores in the maternal-child unit. The mean score for "preparing/carrying medications" was more favourable in the critical care and maternal-child units and less favourable in emergency, medical and surgical units.

\section{Errors related to transcribing were the most frequently reported, followed by errors involving legibility and distractions.}

The top three perceived sources of errors were transcribing (processing), legibility (prescribing) and distractions (administering) (Table 3). For the category "administering," there were no significant differences for results between patient care units (Table 4). However, for perceived transcribing errors (processing), the maternal-child unit responded more favourably than did the medical and surgical units. The perception of legibility (prescribing) being an important source of error was significantly stronger in the emergency unit than in the medical units. As shown in Table 4 , the perception that medication processing and prescribing were sources of errors was associated with patient care units. After applying the Bonferroni correction, the maternal-child unit had a significantly lower mean score on medication processing than did the medical and surgical units. The medical unit had a significantly lower mean score on medication prescribing than did the emergency unit. Years of nursing experience was not associated with perceived sources of error.

\section{Discussion}

This cross-sectional survey among nurses in a university-affiliated general community hospital investigated self-reported medication practices and sources of error. Using sub-scales and items 
Table 2. Results of medication report practices outcomes by unit and nurse experience

\begin{tabular}{|c|c|c|c|c|c|c|c|c|c|}
\hline & \multirow[b]{2}{*}{$\mathbf{n}$} & \multicolumn{2}{|c|}{$\begin{array}{l}\text { Sub-scale 1: Dosage } \\
\text { Care }\end{array}$} & \multicolumn{2}{|c|}{$\begin{array}{l}\text { Sub-scale 2: Right } \\
\text { Patient Identification }\end{array}$} & \multicolumn{2}{|c|}{$\begin{array}{l}\text { Sub-scale 3: } \\
\text { Preparing/Carrying } \\
\text { Medications }\end{array}$} & \multicolumn{2}{|c|}{$\begin{array}{l}\text { Sub-scale 4: } \\
\text { Reporting Errors }\end{array}$} \\
\hline & & $\begin{array}{l}\text { Mean } \\
(S D)^{*}\end{array}$ & p Valuet & $\begin{array}{l}\text { Mean } \\
(S D)^{*}\end{array}$ & p Valuet & $\begin{array}{l}\text { Mean } \\
(\mathrm{SD})^{*}\end{array}$ & p Valuet & $\begin{array}{l}\text { Mean } \\
(\mathrm{SD})^{*}\end{array}$ & p Valuet \\
\hline Overall (N) & 144 & $2.2(1.0)$ & & $2.0(0.8)$ & & $3.5(1.4)$ & & $1.7(0.9)$ & \\
\hline Unit & & & $<.001$ & & $<.001$ & & $<.001$ & & .199 \\
\hline Critical care & 14 & $3.0(1.3)$ & & $2.2(0.8)$ & & $1.8(1.3)$ & & $1.4(0.6)$ & \\
\hline Emergency & 22 & $2.8(1.0)$ & & $1.6(0.6)$ & & $3.5(1.4)$ & & $1.8(1.2)$ & \\
\hline Medicine & 40 & $2.3(0.7)$ & & $1.6(0.5)$ & & $4.3(1.0)$ & & $1.9(1.0)$ & \\
\hline Maternal-child & 29 & $1.4(0.6)$ & & $2.7(0.6)$ & & $2.8(1.4)$ & & $1.4(1.2)$ & \\
\hline Surgery & 15 & $1.6(0.6)$ & & $1.5(0.5)$ & & $4.3(1.0)$ & & $1.9(0.8)$ & \\
\hline Others $\ddagger$ & 24 & $2.7(1.1)$ & & $2.6(1.0)$ & & $3.8(1.2)$ & & $1.5(0.7)$ & \\
\hline Years of experience & & & .066 & & .432 & & .819 & & .579 \\
\hline$<5$ & 38 & $1.9(1.0)$ & & $1.8(0.6)$ & & $3.8(1.4)$ & & $1.8(1.0)$ & \\
\hline$>5$ & 106 & $2.4(1.0)$ & & $2.1(0.9)$ & & $3.4(1.4)$ & & $1.6(0.9)$ & \\
\hline
\end{tabular}

${ }^{*}$ All the outcomes scores are between 1 (most favourable) and 5 (least favourable). †Two-way analysis of variance. $\neq$ Dialysis, mental health, family medicine, long-term care or oncology.

assessing different aspects of practice (dosage care, right patient, preparing/carrying medications and reporting errors) we were able to identify areas where practices may need improvement. Errors related to medication processing (transcribing) were the most frequently reported, followed by errors involving prescribing (legibility) and administering medications (distractions). Dispensing errors were the least frequent. This ranking of perceived causes of error differed minimally from results in the Mayo and Duncan (2003) study, where illegible handwriting (prescribing) ranked the highest, followed by distraction (administration).

Differences among patient care units were found both in medication practices and perceived sources of error. These are likely explained by differences in the patient population and the types and number of medications used on particular patient care units. For example, the maternal-child care unit uses fewer medications than do other patient care units, with more standardized medications being prescribed. Patients within the maternal-child care unit are also less acute and, generally, a healthier population. These factors probably account for the more favourable scores on "dosage care." Maternal-child care units seldom use insulin or heparin. Interestingly, on some patient care units where nurses were more likely to prepare more than one patient medication at the same time, they took greater safety measures for patient identification. Notably, no relation- ships were found between medication practices or perceived sources of error by years of experience. This result is similar to results in both the Mayo and Duncan (2003) and the Osborne et al. (1999) studies, where no significant differences were noted in perceptions related to age and years of practice and medication errors. This study highlights the need for ongoing education programs on medication safety for all nurses, regardless of years of experience.

Since the administration of this survey, numerous measures have been put in place to facilitate medication administration and to reduce the probability of incidents or accidents involving the medication administration process. These include the generalized use of the seven-day computerized medication administration record (MAR) on almost all in-patient care units, with the exceptional use of the 24-hour MAR on the critical care and surgical units. In 2006-2007, 282,740 new orders were automatically transcribed through the use of the computerized MAR, thereby eliminating manual transcriptions by nurses of medication orders. The use of mediplan ${ }^{\circledR}$, used by nurses, on the patient care units and the medipharm ${ }^{\circledR}$ computerized medication reordering processes, used by pharmacists, has eliminated the legibility concerns for reorders. However, legibility concerns remain for new orders, which continue to be handwritten. Since 2006, intravenous heparin solution bags have been sent preprepared from the pharmacy to the patient care units. 
Table 3. Proportion of medication errors, per nurse, per category $(N=144)$

\begin{tabular}{|c|c|c|c|}
\hline Error and Items & Rank & $\mathbf{n}$ & $\%$ \\
\hline \multicolumn{4}{|l|}{ Processing } \\
\hline Processing: transcription error & 1 & 106 & 73.6 \\
\hline Processing: no order sent to/received by pharmacy & 2 & 60 & 41.7 \\
\hline Processing: error checking MAR/medication sheet & 3 & 57 & 39.6 \\
\hline Processing: computer entry error & 4 & 38 & 26.4 \\
\hline \multicolumn{4}{|l|}{ Mean proportion of processing errors $=0.45(S D=0.27)^{*}$} \\
\hline \multicolumn{4}{|l|}{ Prescribing } \\
\hline Prescribing: legibility & 1 & 71 & 49.3 \\
\hline Prescribing: confusing order/instructions & 2 & 68 & 47.2 \\
\hline Prescribing: incorrect order & 3 & 27 & 18.8 \\
\hline Prescribing: misunderstood verbal order & 4 & 19 & 13.2 \\
\hline \multicolumn{4}{|l|}{ Mean proportion of prescribing errors $=0.32(\mathrm{SD}=0.26)^{*}$} \\
\hline \multicolumn{4}{|l|}{ Administering } \\
\hline Administering: distractions & 1 & 81 & 56.3 \\
\hline Administering: miscalculation & 2 & 49 & 34.0 \\
\hline Administering: procedure/policy not followed & 3 & 25 & 17.4 \\
\hline Administering: knowledge deficit & 4 & 19 & 13.2 \\
\hline \multicolumn{4}{|c|}{ Mean proportion of administering errors $=0.30(S D=0.23)^{*}$} \\
\hline \multicolumn{4}{|l|}{ All dispensing } \\
\hline Dispensing: labels of medications look alike & 1 & 19 & 13.2 \\
\hline Dispensing: names of medications look alike & 2 & 17 & 11.8 \\
\hline Dispensing: mislabelled & 3 & 16 & 11.1 \\
\hline Dispensing: filled incorrectly & 4 & 15 & 10.4 \\
\hline Mean proportion of dispensing errors $=0.12$ (SD $=0$ & & & \\
\hline
\end{tabular}

$M A R=$ medication administration record.

${ }^{*}$ Adjusting for the total numbers of opportunity. practices and review related policies and procedures as well as the incident/accident reporting process for medication errors. Policies and procedures were revised to better address safety issues in medication administration.

Furthermore, the previously existing mentorship program for the training of new nurses was standardized and improved to include an evaluation of the new employees' knowledge of the medication administration procedures. This allowed for a tailoring of the mentorship to each individual's needs while providing comprehensive training covering the essential elements common to all areas.

Frequent in-service education sessions are being offered to all nurses on all shifts to encourage reporting of healthcare servicesrelated incidents and accidents, possibly explaining the favourable scores overall on "error reporting." The systems approach to reducing errors was introduced at St. Mary's in 2002 and has been found to be an effective means to encourage incident/accident reporting.

\section{Limitations}

There were two identified limitations to this study. The first limitation was the grouping of practice experience into the broad categories of less than or more than five years, originally designed to ensure anonymity. A more graduated scale with a larger sample size to maintain anonymity may be more sensitive and helpful in determining the impact of practice experience on perceptions and actual practice.

The second limitation involved the use of a questionnaire survey which may have had an impact on the reporting of current practices and error reporting behaviours (sub-scales one to four). Direct observation has been found to be a more sensitive method for detecting medication administration behaviours. A subsequent study of medication administration practices comparing perceptions with observed behaviours and actual reported incidents may yield a more accurate portrait of current practices.

\section{Conclusions}

In spite of the limitations outlined above, this study demonstrates the need for more education for all nurses, taking into consideration the role adjustment period for new nurses as well as the time associated with preceptorship programs. The hensive booklet titled Medication Administration (phases I and II). Both documents reinforce safe medication administration 
Table 4. Results of perceived sources of error outcomes by unit and nurse experience

\begin{tabular}{|c|c|c|c|c|c|c|c|c|c|}
\hline & \multirow[b]{2}{*}{$\mathbf{n}$} & \multicolumn{2}{|c|}{ Processing } & \multicolumn{2}{|c|}{ Prescribing } & \multicolumn{2}{|c|}{ Administering } & \multicolumn{2}{|c|}{ Dispensing } \\
\hline & & $\begin{array}{l}\text { Mean } \\
(S D)^{*}\end{array}$ & p Valuet & $\begin{array}{l}\text { Mean } \\
(S D)^{*}\end{array}$ & p Valuet & $\begin{array}{l}\text { Mean } \\
\text { (SD)* }\end{array}$ & p Valuet & $\begin{array}{l}\text { Mean } \\
\text { (SD)* }\end{array}$ & p Valuet \\
\hline Overall (N) & 144 & $0.45(0.3)$ & & $0.32(0.3)$ & & $0.30(0.2)$ & & $0.12(0.2)$ & \\
\hline Unit & & & .001 & & .0002 & & .049 & & .624 \\
\hline Critical care & 14 & $0.33(0.2)$ & & $0.30(0.2)$ & & $0.38(0.2)$ & & $0.16(0.2)$ & \\
\hline Emergency & 22 & $0.39(0.2)$ & & $0.51(0.3)$ & & $0.27(0.3)$ & & $0.09(0.1)$ & \\
\hline Medicine & 40 & $0.54(0.3)$ & & $0.23(0.2)$ & & $0.23(0.2)$ & & $0.13(0.2)$ & \\
\hline Maternal-child & 29 & $0.34(0.2)$ & & $0.39(0.2)$ & & $0.33(0.2)$ & & $0.15(0.2)$ & \\
\hline Surgery & 15 & $0.57(0.3)$ & & $0.33(0.2)$ & & $0.25(0.2)$ & & $0.12(0.2)$ & \\
\hline Others $\ddagger$ & 24 & $0.51(0.3)$ & & $0.23(0.3)$ & & $0.41(0.3)$ & & $0.06(0.13)$ & \\
\hline Years of experience & & & .067 & & .912 & & .923 & & .524 \\
\hline$<5$ & 38 & $0.42(0.3)$ & & $0.30(0.2)$ & & $0.29(0.3)$ & & $0.13(0.2)$ & \\
\hline$>5$ & 106 & $0.46(0.3)$ & & $0.33(0.3)$ & & $0.31(0.2)$ & & $0.11(0.2)$ & \\
\hline
\end{tabular}

${ }^{*}$ All the outcomes scores (rate) are between 0 and 1 (higher: more sources of error).

†Two-way analysis of variance.

‡Dialysis, mental health, family medicine, long-term care or oncology.

authors acknowledge that more research is needed on the impact of senior nurses, preceptorship programs and patient care unit safety culture on the medication safety practices of junior nurses. Future research should use a more graduated scale for the years of experience and an observational approach to error reporting and practice behaviours.

\section{About the Authors}

Markirit Armutlu is the quality and risk management coordinator at St. Mary's Hospital Center, Montreal, Quebec. You can contact her at 514-345-3511, ext. 6586, by fax at 514-734-2636 or by email at markirit.armutlu@ssss.gouv.qc.ca.

Mary-Lou Foley is a liaison nurse with the programs of medicine, surgery and mental health at St. Mary's Hospital Center.

Judy Surette is a quality analyst in the Quality Assessment Unit at St. Mary's Hospital Center.

Eric Belzile is a statistician in the Department of Clinical Epidemiology and Community Studies at St. Mary's Hospital Center.

Jane McCusker is director of the Department of Clinical Epidemiology and Community Studies at St. Mary's Hospital Center.

\section{Acknowledgements}

The authors gratefully acknowledge the following contributors to this article: Suzanne Dehaney (student, data analyst),
Nathalie Beaudoin (assistant to the vice-president of operations and nursing), Marie Iskandar (chief pharmacist), Linda Cardinal (quality assessment analyst), Judith Sandhurst (family physician) and Linda Bambonye (vice-president of operations and nursing).

The authors also wish to acknowledge the invaluable assistance of the Quality Assessment Unit and the Department of Clinical Epidemiology and Community Studies in supporting this study. HQ

\section{Bibliography}

Anderson, D.J. and C.S. Webster. 2001. "A Systems Approach to the Reduction of Medication Error on the Hospital Ward." Nursing and Health Care Management Issues - Journal of Advanced Nursing 35(1): 34-41.

Casey, K., R. Fink, M. Frugman and J. Propst. 2004. "The Graduate Nurse Experience." The Journal of Nursing Administration 34(6): 30311 .

Cohen, H., E.S. Robinson and M. Mandrack. 2003. "Getting to the Root of Medication." Nursing 33(9): 36-45.

Cohen, M.R. 1999. "Causes of Medication Errors.” In M.R. Cohen, ed., Medication Errors. Washington, DC: American Pharmacists Association.

Duchscher Boychuk, J.E. and L.S. Cowin. 2006. “The New Graduate's Professional Inheritance.” Nursing Outlook 54(3):152-8.

Haw, C., J. Stubbs and G. Dickens. 2007. "An Observational Study of Medication Administration Errors in Old-age Psychiatric Inpatients." International Journal of Quality in Health Care 19(4): 210-16. 
Hicks, R.W., S.C. Becker, D. Krenzischeck and S.C. Beyea. 2004. "Medication Errors in the PACU: A Secondary Analysis of MEDMARX Findings." Journal of PeriAnesthesia Nursing 19(1): 18-28.

Huber, D. 2002. "Nevada Nurses Participate in Medication Errors Survey." Nevada RN Information 11(3): 18-9.

Kazaoka, T., K. Ohtsuka, K. Ueno and M. Mori. 2007. "Why Nurses Make Medication Errors: A Simulation Study." Nurse Education Today 27(4): 312-7.

Kohn, L.T., J.M. Corrigan and M.S. Donaldson, eds. 1999. To Err Is Human: Building a Safer Health System. Washington, DC: National Academy Press.

Leape L.L., D.W. Bates, D.J. Cullen, J. Cooper, H.J. Demonaco, T. Gallivan, R. Hallisey, J. Ives, N. Laird and G. Laffel. 1995. "Systems Analysis of Adverse Drug Events. ADE Prevention Study Group." Journal of the American Medical Association 274: 35-43.

Manun'Ebo, M., S. Cousens, P. Haggerty, M. Calengaie, A. Ashworth and B. Kirkwood. 1997. "Measuring Hygiene Practices: A Comparison of Questionnaires with Direct Observations in Rural Zaire.” Tropical Medicine and International Health 2(11): 1015-21.

Mayo, A.M. and D. Duncan. 2004. "Nurse Perception of Medication Errors. What We Need to Know for Patient Safety." Journal of Nursing Care Quality 19(3): 209-17.

McBride-Henry, K. and M. Foureur. 2006. "Medication Administration Errors: Understanding the Issues." Australian Journal of Advanced Nursing 23(3): 33-41.

Nicholson, D., W. Hersh, T.K. Gandhi, S.N. Weingart and D.W. Bates. 2006. "Medication Errors: Not Just a Few Bad Apples." Journal of Clinical Outcomes Management 13(2): 114-5.

Osbourne, J., K. Blias and J. Hayes. 1999. "Nurses' Perceptions: When Is It a Medication Error?" Journal of Nursing Administration 29(4): 33-38.

O’Shea, E. 1999. "Factors Contributing to Medication Errors: A Literature Review." Journal of Clinical Nursing 8(5): 496-504.

Sahlberg-Blom, E., B.-M. Ternestedt and J.-E. Johansson. 2001. "Is Good 'Quality of Life' Possible at the End of Life? An Explorative Study of the Experiences of a Group of Cancer Patients in Two Different Care Cultures." Journal of Clinical Nursing 10(4): 550-62.

Sim, T.A. and J. Joyner. 2002. "A Multidisciplinary Team Approach to Reducing Medication Variance." Joint Commission Journal on Quality Improvement 28(7): 403-9.

Song, Y. and G.-J. Knapp. 2007. "Quantitative Classification of Neighborhoods: The Neighborhoods of New Single-Family Homes in the Portland Metropolitan Area." Journal of Urban Design 12(1): $1-24$

Spouce, J. 2001. "Bridging Theory and Practice in the Supervisory Relationship: A Sociocultural Perspective." Journal of Advanced Nursing 33(4): 512-22.

Stewart, B.M. and L.E. Krueger. 1996. "An Evolutionary Concept Analysis of Mentoring in Nursing." Journal of Professional Nursing 12(5): 311-21.

Thomka, L.A. 2001. “Graduate Nurses' Nurses' Experiences of Interactions with Professional Nursing Staff during Transition to the Professional Role." The Journal of Continuing Education in Nursing 32(1): 15-9.

Thomka, L.A. 2007. "Mentoring and Its Impact on Intellectual Capital: Through the Eyes of the Mentee." Nursing Administration Quarterly 31(1): 22-6.
Winson, G. 1991. "A Survey of Nurse's Attitudes towards Single Administration of Medications." Nursing Practice 4(3): 20-3.

\section{Appendix A}

Information on the usual patient care unit where the nurse worked and years of practice (five years or less, and more than five years) was collected. Principal components analysis was used to reduce the questions into a smaller number of sub-scales that account for most of the variance in the data. All components associated with an eigenvalue greater than one were first selected. A varimax rotation was then applied to the chosen components to obtain more interpretable sub-scales (Stevens 1996). Questions with the greatest factor loadings, $>0.50$, and in the same direction, were included in each sub-scale. The internal consistency of each sub-scale thus derived was estimated using Cronbach's alpha statistic. The score for each sub-scale was estimated as the average score for that sub-scale.

Both mean sub-scale scores of medication practice behaviours and mean rates of perceived sources of medication errors were compared across categories for two occupational characteristics (years of experience and work unit) using two-way analysis of variance (ANOVA) models. Patient care units were coded according to the following categories: critical care, emergency, medicine, maternal-child, surgery and other (units with seven or fewer respondents, including mental health, dialysis, oncology, family medicine and long-term care). The interaction between years of experience and work unit was tested and found not significant (using alpha of .1) for all two-way ANOVA models. Bonferroni contrasts were used to compare units. All analyses were performed using the SAS 9.1 software package (SAS 2007). In the interpretation, the $p$ value of a two-sided test was considered statistically significant when less than .05 .

\section{References}

SAS Institute Inc. 2007. SAS Online Doc, Version 9.1. Cary, NC: SAS Institute Inc. Retrieved September 3, 2007. <http://www.sas.com>.

Stevens, J.P. 1996. Applied Multivariate Statistics for the Social Sciences. Hillsdale, NJ: Lawrence Erlbaum Associates. 